Throughout, Carter balances between her insistence that the Catholic Reformation was not a top-down process and an organizational structure that is largely top down. The Catholic Reformation, and the religious education that was its centrepiece, was not simply an act of state but the result of collaboration and negotiations between bishops, priests, teachers, and villagers. This said, the book starts with the educational goals of Tridentine reformers and argues for the successful implementation of these goals in the villages. I found myself imagining a second book that complements this one by beginning not with the Catechism of the Council of Trent, but with village parents. From the perspective of the bishops, the education Carter studies looks partially successful - even after 200 years. From the perspective of parents, welcoming schoolmasters into their towns to train their children to be good Catholics (in the more practical sense), Carter's optimism seems perhaps even more justified.

In sum, Karen Carter's Creating Catholics offers much more than its title might imply, and will be of interest to readers interested in topics ranging from the Reformation to the French Revolution, and from the history of childhood and education to the history of state building. It makes a fine contribution to early modern European history overall.

JESSe Spohnholz, Washington State University

\title{
Dauvois, Nathalie.
}

La vocation lyrique. La poétique du recueil lyrique en France à la Renaissance et le modèle des Carmina d'Horace.

Paris: Éditions Classiques Garnier, 2010. 263 p. ISBN 978-2-8124-0104-6 (broché) $39,55 €$

Nathalie Dauvois est déjà connue comme experte du lyrisme de la Renaissance, ayant publié des livres à ce sujet en 2000 (Le sujet lyrique à la Renaissance, Presses Universitaires de France) et en 2007 (La Renaissance de l'Ode : L'ode française au tournant des années 1550, Honoré Champion). Elle livre ici une étude du lyrisme fondée sur une définition générique bornée par une forme poétique particulière. En effet, qui dit poésie lyrique à la Renaissance dit ode, et plus précisément un recueil d'odes imitant le modèle antique. C’est Horace 
en particulier qui est préféré chez les Français, poète lyrique singulier dans son rôle d'imitateur lui-même des poètes grecs et surtout dans sa pérennité, à la différence de Sappho, Pindare et Anacréon dont les odes n'ont survécu qu'en fragments. Il ne s'agit donc pas encore de la définition qui nous sera fournie par l'époque romantique, ce que l'auteure ne manque pas de souligner au seuil de son ouvrage (p. 9-10). Pour les poètes français des années 1550, il est surtout question d'un lyrisme générique, ainsi seuls les livres de carmina inspirées d'Horace sont considérés comme des exercices lyriques. C'est cette prolifération, d'une durée relativement courte, dont traite Dauvois, qui prend comme point de départ les Vers lyriques de Jacques Peletier du Mans (1547) et considère d'autres recueils publiés en France jusqu'à la fin de la décennie suivante.

Après avoir défini son objectif dans l'« Introduction », elle présente dans le « Préambule » le contexte historique du Moyen Âge tardif qui précède l'élan français de l'humanisme européen. Elle tâche d'examiner le rôle du lyrisme dans la poésie française, avant que la Pléiade ne revendique l'imitation des Anciens et que l'ode ne s'impose, au détriment des formes médiévales. Dans le chapitre suivant, " Héritages et ruptures ", elle continue à énumérer les précurseurs du lyrisme de l'ode horacienne, identifiant des modèles italiens (p. 40-50), aussi bien que français (p. 51-64).

Une fois le contexte historique établi, Dauvois passe à une analyse du lyrisme humaniste en France, examinant les différents aspects de la poétique du recueil d'odes. Elle commence par un chapitre sur la "Varietas », principe prôné par les humanistes, surtout suite aux maintes éditions commentées des Carmina d'Horace à la fin du quinzième siècle (p. 71). Il s'agit d'une variété dans les sujets des odes aussi bien que dans leurs formes, qui distinguent l'ode de l'autre forme dominante de cette époque, le sonnet (p. 100). Suit une analyse des "Performance et pragmatique lyriques », dans laquelle l'auteure met l'accent sur le caractère performatif de l'ode à la Renaissance, trait qui non seulement remonte au modèle horacien mais qui suggère également ses origines dans « une poésie cérémonielle, liée aux événements du culte, chantée le plus souvent par un chœur, dont les fonctions se diversifient progressivement " (p. 106). Dans le chapitre intitulé « Passions », Dauvois souligne l'expression des sentiments comme liant la poésie lyrique à la musique - rapport qui rappelle encore une fois ses origines dans la performance (p. 139-141) - en précisant que dans le modèle horacien, c'est la modération ou la tempérance 
qui domine (p. 152 et 158), tempérance reflétée dans la forme jusqu’au mètre (p. 153). Elle consacre son chapitre «Strophes» à un examen de ces schémas métriques. Enfin le chapitre «Voix » aborde les voix du lyrisme, car la poésie de la Renaissance comprend non seulement la voix du je émotif, mais également la voix « officielle » et « collective » inspirée des Carmina d'Horace (p. 231).

Malgré l'efficacité d'une telle organisation pour tracer cette définition particulière du lyrisme, certains sujets ne se prêtent pas facilement à cette structure. Notamment, selon l'auteure, les tragédies de l'époque fournissent elles aussi des exemples de poésie lyrique dans les paroles des chœurs, qui sont organisées sous «la forme d'odes pindariques» (p. 118). Elle entame cette analyse dans la troisième section, "Performance et pragmatique lyriques ", en mentionnant brièvement quelques exemples avant de conclure : "On pourrait analyser les chœurs des tragédies de cette première renaissance de la tragédie comme autant d'odes rendues par le contexte dramatique et la représentation à leur performantialité...» (p. 119). Elle y revient dans la dernière section consacrée à une caractéristique du lyrisme, "Voix », où son analyse devient plus détaillée (p. 220-227). La question de l'ode dans le théâtre est intéressante, surtout pour son rapport à cette " performantialité ». Elle permet en effet d'évoquer le contexte de l'origine antique de la poésie lyrique, toutefois, l'organisation de l'étude de Dauvois rend son analyse de ce phénomène culturel assez peu clair. De plus, cette partie semble constituer plutôt une digression qu'un élément à l'appui de son argumentation, qui insiste autant sur une définition du lyrisme contrainte par la forme générique de l'ode et par le rassemblement de poésies dans un ensemble livresque.

Néanmoins, dans l'ensemble, l'ouvrage de Dauvois donne une présentation organisée et érudite du lyrisme horacien en France au seizième siècle. Dans le sillage de l'étude prééminente de Carol Maddison sur l'ode en Europe à la Renaissance (Apollo and the Nine: A History of the Ode, 1960), elle se concentre sur la naissance de l'ode vernaculaire en France. L'auteure se sert surtout des vers de la Pléiade pour illustrer cette forme, avec une attention particulière prêtée à Peletier du Mans et à Ronsard. Elle se limite à cette génération, notant que le contexte des guerres de religion exigeait d'autres formes poétiques. Même si l'on continuait à faire des odes, moins nombreux sont les auteurs qui en publiaient des recueils après 1560 (p. 206). Ainsi, l'on peut comprendre pourquoi elle ne traite pas de poètes tels Amadis Jamyn, disciple de Pierre de Ronsard, qui insère des odes dans ses recueils publiés dans les années 1570. 
L'ode vernaculaire en France connut sa plus grande gloire chez les poètes humanistes de la Renaissance, qui lui consacrèrent « [un] élan et [une] énergie collective» (p. 237), ne serait-ce que pendant une très courte durée.

ANNICK MACASKILL, University of Western Ontario

\section{Goldthwaite, Richard A.}

\section{The Economy of Renaissance Florence.}

Baltimore: The Johns Hopkins University Press, 2009. Pp. xviii, 649. ISBN 9780-8018-8982-0 (hardcover) \$57.

Following hard on the heels of John Najemy's magisterial History of Florence, 1200-1575 (2006), Richard Goldthwaite's monumental synthesis aims to compensate for the relative neglect of the city's economic vis-à-vis its political and cultural life; after all, there can be no question that it was the - by any contemporary standard - extraordinary wealth generated by the Florentine banking and textile sectors that underwrote the city's cultural monuments and republican culture, both of which have long been the privileged focus of scholarly attention. The fruit of an unrivalled intimacy with medieval and Renaissance business records (gained over more than four decades of research in Florentine collections) and an encyclopedic knowledge of Italian and English scholarship, this book is essential reading not only for economic historians, whom it directs to fruitful new avenues of research, but also for historians of Renaissance Florence generally, who will gain insight into the material basis of phenomena such as art, politics, and ideology

Goldthwaite divides his treatment into two parts that deal in turn with the global context of the Florentine economy and close studies of the local, urban economy, the whole prefaced by a lucid prehistory tracing the rise of Florence, against all geographical odds, to fourteenth-century predominance. Neither a port, like Genoa, Venice, or Pisa, nor, like Siena and Lucca, a waystation on the major routes to Rome, Florence was ill-favoured to become a financial or mercantile giant; indeed, she was outstripped in the early phases of the "commercial revolution" by all five, and was never an international emporium on the scale of Antwerp, Lyons, or Venice. Nevertheless, by the first quarter 